\title{
Luteolin suppresses the metastasis of triple-negative breast cancer by reversing epithelial-to-mesenchymal transition via downregulation of $\beta$-catenin expression
}

\author{
DAN LIN ${ }^{1,2 *}$, GE KUANG ${ }^{2 *}$, JINGYUAN WAN $^{2}$, XIANG ZHANG ${ }^{1}$, \\ HONGZHONG $\mathrm{LI}^{3}$, XIA GONG ${ }^{4}$ and HONGYUAN LI ${ }^{1}$ \\ ${ }^{1}$ Department of Endocrine and Breast Surgery, The First Affiliated Hospital of Chongqing Medical University; \\ ${ }^{2}$ Chongqing Key Laboratory of Biochemistry and Molecular Pharmacology, Chongqing Medical University; \\ ${ }^{3}$ Molecular Oncology and Epigenetics Laboratory, The First Affiliated Hospital of Chongqing Medical University; \\ ${ }^{4}$ Department of Anatomy, Chongqing Medical University, Chongqing 400016, P.R. China
}

Received May 23, 2016; Accepted August 23, 2016

DOI: $10.3892 /$ or.2016.5311

\begin{abstract}
The metastasis of breast cancer is associated with dismal prognosis and high mortality due to the lack of effective treatment. Luteolin, a natural flavonoid compound, has been shown to exert antitumor activity in various types of cancers. However, the effects and mechanisms of luteolin on the metastasis of triple-negative breast cancer (TNBC) remain elusive. In the present study, we found that pretreatment of highly metastatic TNBC cell lines with luteolin dose-dependently inhibited cell migration and invasion, and reversed epithelialmesenchymal transition (EMT) as determined by altered morphological characteristics, downregulated epithelial markers and upregulated mesenchymal markers, and inhibited EMT-related transcription factors. In an in vivo metastasis experiment using a xenograft model, luteolin markedly inhibited lung metastases of breast cancer and the expression of EMT molecules vimentin and Slug in primary tumor tissues. Notably, luteolin also suppressed the expression of $\beta$-catenin mRNA and protein in vitro and in vivo. Furthermore, overexpression of $\beta$-catenin by adenoviruses blocked these benefits of luteolin on invasion and metastases of breast cancer. In conclusion, all these results indicated that luteolin
\end{abstract}

Correspondence to: Dr Hongyuan Li, Department of Endocrine and Breast Surgery, The First Affiliated Hospital of Chongqing Medical University, 1 Youyi Road, Chongqing 400016, P.R. China

E-mail: hongy_li@hotmail.com

Dr Xia Gong, Department of Anatomy, Chongqing Medical University, 1 Yixueyuan Road, Yuzhong, Chongqing 400016, P.R. China

E-mail: xiagong5@hotmail.com

${ }^{*}$ Contributed equally

Key words: luteolin, cancer metastasis, triple-negative breast cancer, epithelial-to-mesenchymal transition, $\beta$-catenin effectively suppressed metastases of breast cancer by reversing EMT, which may be mediated by downregulation of $\beta$-catenin.

\section{Introduction}

Breast cancer is the second leading cause of cancer-related deaths, and the most common cancer among women worldwide (1). Although numerous measures and drugs have been applied to the clinic, metastasis is still one of the most critical issues in patients with breast cancer (2). Therefore, the identification of effective drugs and exploring the mechanisms which suppress breast cancer metastasis may provide hope to clinical therapies.

The metastasis of cancer cells are reminiscent of epithelial-mesenchymal transition (EMT) usually occurring in embryonic development, tissue repair and tumor progression $(3,4)$. During the EMT process, epithelial-like cancer cells lose cell-cell contacts and acquire mesenchymal properties, which are believed to help cancer cells to gain the abilities of migration and invasion, resulting in the disassociation of cancer cells from the primary tumor and migration to distant organs. From a molecular viewpoint, EMT is characterized by downregulation of epithelial cell markers such as E-cadherin and claudin, and upregulation of mesenchymal molecules such as vimentin and $\mathrm{N}$-cadherin $(5,6)$. Several transcriptional factors, including Snail, Slug, ZEB and Twist, have been found to be involved in the regulation of EMT $(6,7)$. These transcription factors directly or indirectly repress the transcriptional expression of E-cadherin, resulting in the loss of epithelial markers and the acquisition of mesenchymal features (3). Recent studies have also suggested that EMT is connected with the acquisition of cancer stem cell properties, development of drug resistance, and induction of angiogenesis, providing various distinct benefits to tumor progression (8-12).

Supporting evidence from epidemiology indicates that some natural dietaries may exhibit beneficial actions against the risk or progression of breast cancer (13). Luteolin (Fig. 1A), one of the natural flavonoid compounds found in many plants such as carrots, celery, broccoli, perilla leaf and seed, has 
been reported to possess many biological properties such as anti-inflammation, anti-allergy, antioxidant, anticancer and anti-microbial (14-16). Previous studies indicate that luteolin exhibits a wide range of antitumor activities in various types of cancers by inhibiting cell proliferation and tumor growth, promoting cancer cell apoptosis and cell cycle arrest, sensitizing drug resistance, and mitigating invasiveness and metastasis of cancer cells $(14,17-19)$. Specifically, it has been found that luteolin enhanced paclitaxel-induced apoptosis in human breast cancer (20), and sensitized drug-resistant human breast cancer cells to tamoxifen (21). Furthermore, luteolin effectively blocked progestin-dependent angiogenesis and the stem cell-like phenotype in human breast cancer cells (22). Although these studies revealed its protective roles in malignancy, the effects and underlying mechanisms of luteolin on the metastasis of highly aggressive triple-negative breast cancer (TNBC) remain largely unexplored.

In the present study, we chose two TNBC cell lines MDA-MA-231 and BT5-49, which have been indicated to possess highly malignant transfer traits $(23,24)$, to investigate the potential functions and mechanisms of luteolin on the metastasis of TNBC in vitro and in vivo.

\section{Materials and methods}

Reagents and antibodies. Luteolin (purity $>99 \%$ ) was obtained from Pulus Biology Technology (Chengdu, China). RNA isolation kit, PrimeScript RT and PCR reagent kits were purchased from Takara (Dalin, China). Matrigel and Transwell chambers were obtained from Corning (Corning, NY, USA). Antibodies used in the present study were purchased from the following sources: anti-E-cadherin, anti-claudin, anti-vimentin, anti$\mathrm{N}$-cadherin, anti-Snail, anti-Slug, anti-ZEB1 and anti- $\beta$-catenin antibodies were from Cell Signaling Technology (Danvers, MA, USA); anti-GAPDH antibody was from Santa Cruz Biotechnology (Santa Cruz, CA, USA); anti-Ki67 was from Abcam (Cambridge, UK); anti-F-actin-Red 555 was from Invitrogen (Carlsbad, CA, USA).

Cell culture and treatment. Human breast cancer cell lines MDA-MB-231 and BT5-49 were obtained from the American Type Culture Collection (ATCC; Manassas, VA, USA) and cultured at $37^{\circ} \mathrm{C}$ in Dulbecco's modified Eagle's medium (DMEM) (HyClone, Los Angeles, CA, USA) with $10 \%$ fetal bovine serum (FBS) (Gibco, Grand Island, NY, USA) and 1\% penicillin/streptomycin in a humidified atmosphere of $5 \%$ $\mathrm{CO}_{2}$. The cells were treated with various concentrations (10, 30 and $100 \mu \mathrm{M}$ ) of luteolin for the indicated time.

Nude mice and in vivo metastasis assay. Female nude mice at 4-5 weeks old following approval by the Animal Ethics Committee of Chongqing Medical University were housed according to the National and Institutional Guidelines for Humane Animal Care in specific pathogen-free (SPF) laboratory animal environmental. For the xenograft metastasis experiment, female nude mice were subcutaneously injected with $1 \times 10^{6}$ MDA-MB-231 cells with or without transduction of Ad-GFP or Ad- $\beta$-catenin, and subjected to intraperitoneal injection of luteolin $(100 \mathrm{mg} / \mathrm{kg})$ three times every week when the tumor reached $1 \mathrm{~cm}^{3}$ in volume. Eight weeks later, the mice were sacrificed under anesthesia. The tumor nodules on the lung were counted. The primary tumor tissues and lung were collected for further analyses.

Scratch migration assay. MDA-MB-231 and BT5-49 cells were seeded into a 6-well plate in culture medium and allowed to grow to $100 \%$ confluence. A sterile toothpick was used to scrap the monolayer cells creating a wound. The scraped cells were washed out with phosphate-buffered saline (PBS), and the remaining cells continued to culture in the absence or presence of different concentration of luteolin for $18 \mathrm{~h}$. The breast cancer cells were observed and the areas of the wound were measured.

Transwell invasion assay. The invasion was measured using the Matrigel-coated 24-well Transwell chamber. Briefly, MDA-MB-231 and BT5-49 cells were trypsinized, washed and seeded into the upper chamber in a serum-free medium at a density of $2.5 \times 10^{5}$ cells/well. The lower chamber contained complete DMEM with 10\% FBS. Following incubation for $8 \mathrm{~h}$, the cells on the Matrigel membrane were fixed and stained using crystal violet. The invaded cells were counted.

Immunofluorescent (IF) staining. For IF analysis, the cells or tissues on the slides were fixed in $4 \%$ paraformaldehyde at room temperature and permeabilized using $0.05 \%$ Triton X-100. Then, the slides were first immersed in blocking solution containing $1 \%$ BSA in PBS, followed by incubation with the primary antibody overnight at $4^{\circ} \mathrm{C}$. The slides were then incubated in PBS buffer with fluorescently labeled secondary antibody for $1 \mathrm{~h}$ at room temperature in the dark. Nuclei were counterstained with 4',6-diamidino-2-phenylindole (DAPI) and observed under a fluorescence microscope (Nikon, Tokyo, Japan).

Protein isolation and western blotting. The cells were lysed in protein lysis buffer to collect total proteins. The concentration of protein was determined using the BCA kit. Total proteins $(40 \mu \mathrm{g})$ were fractionated on $10 \%$ SDS gel, and then transferred to polyvinylidene fluoride (PVDF) membranes. The membranes were blocked with 5\% BSA in Tris-buffered saline (TBS) containing $0.1 \%$ Tween-20, followed by incubation with the primary antibody at $4^{\circ} \mathrm{C}$ overnight. After washing with TBST for three times, the membranes were incubated with secondary antibody conjugated with horseradish peroxidase (HRP). The membranes were visualized using the enhanced chemiluminescent system and short exposure of the membrane to X-ray film.

Quantitative reverse transcription-polymerase chain reaction ( $q R T-P C R)$. Total RNA from the cancer cells was extracted using the RNA isolation kit according to the manufacturer's instructions. Then cDNA was synthesized using PrimeScript RT reagent kit with $1 \mu \mathrm{g}$ total RNA. qPCR was performed using the PCR kit. The following primer sequences were used: E-cadherin sense, 5'-TCCTGGGCAGAGTGAA TTTTGAAGA-3' and antisense, 5'-AAACGGAGGCCTG ATGGGG-3'; claudin sense, 5'-CCTCCTGGGAGTGATAGC AAT-3' and antisense, 5'-GGCAACTAAAATAGCCAGA CCT-3'; vimentin sense, 5'-TACAGGAAGCTGCTGGA 

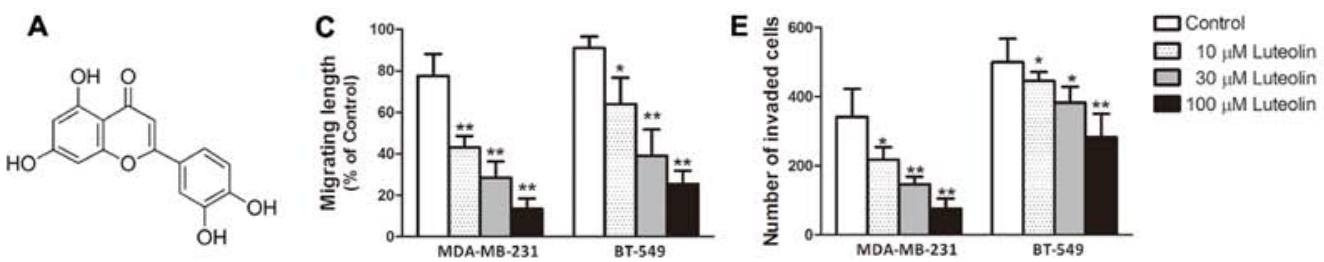

B
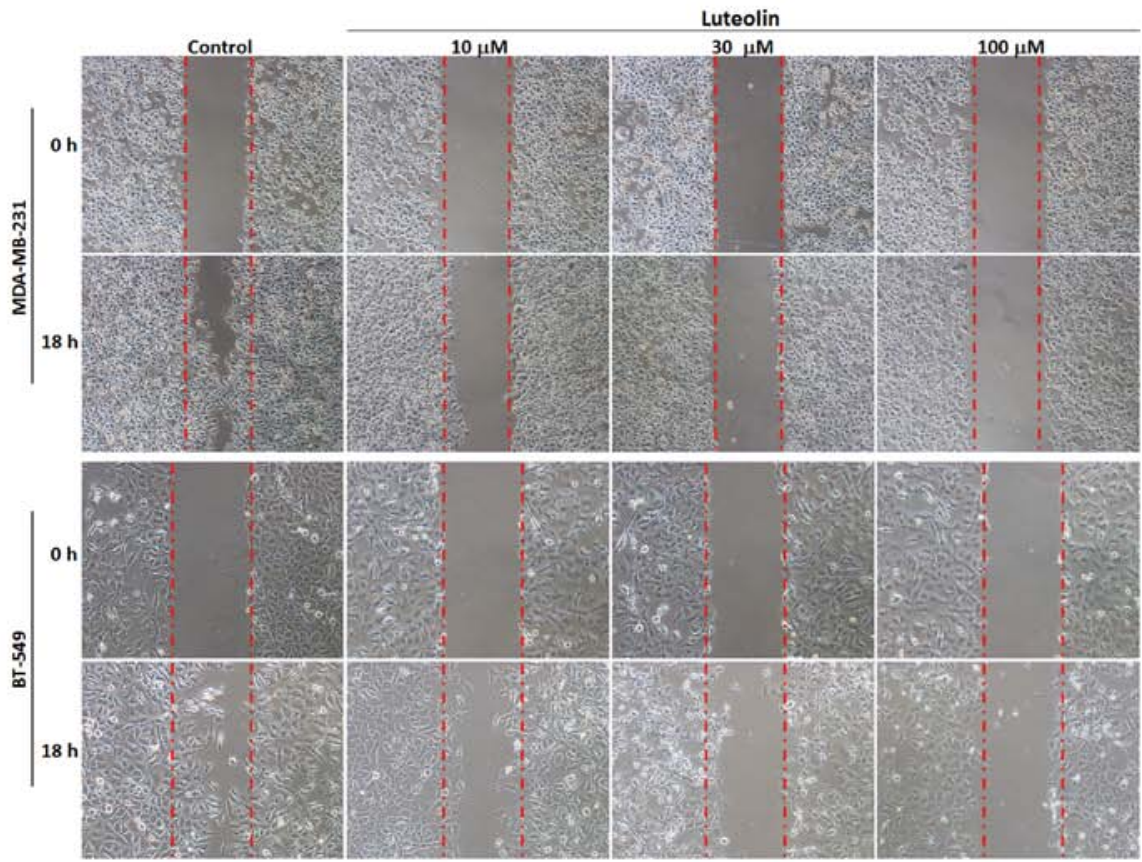

D

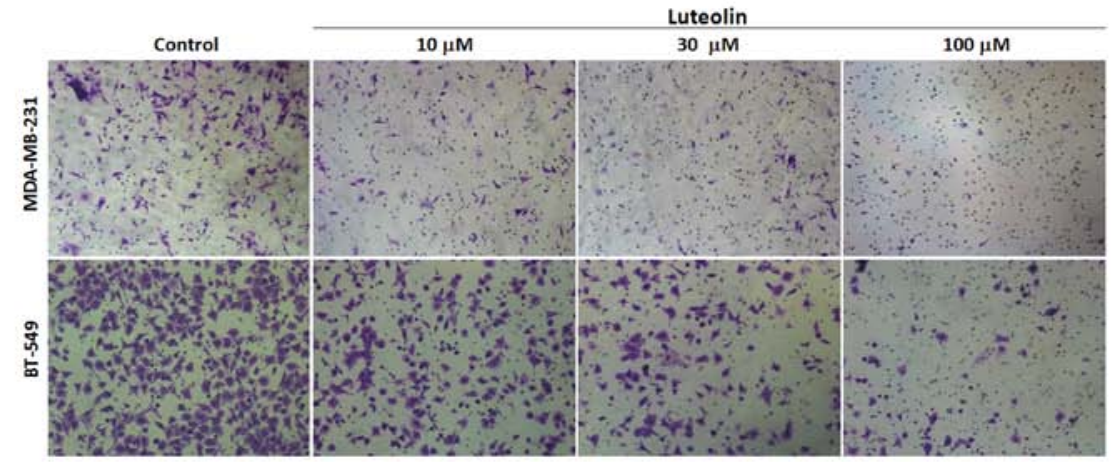

Figure 1. Luteolin inhibits the migration and invasion of breast cancer cells. Triple-negative breast cancer (TNBC) cell lines MDA-MB-231 and BT5-49 were pretreated with various concentrations of luteolin $(10,30$ and $100 \mu \mathrm{M})$ for the indicated time. (A) Chemical structure of luteolin. (B) The cell migration was determined by wound-healing assay at $24 \mathrm{~h}$ after luteolin treatment. (C) The quantification of cell migration. (D) The cell invasion was determined by Transwell invasion assay at $8 \mathrm{~h}$ after luteolin treatment. (E) The quantification of cell invasion. The results are shown as the mean $\pm \mathrm{SD}$ of three experiments; ${ }^{*} \mathrm{P}<0.05$, ${ }^{* *} \mathrm{P}<0.01$ compared with the control.

AGG-3' and antisense, 5'-ACCAGAGGGAGTGAATCCAG-3'; $\mathrm{N}$-cadherin sense, 5'-AGCCAACCTTAACTGAGGAGT-3' and antisense, 5'-GGCAAGTTGATTGGAGGGATG-3'; Snail sense, 5'-TCGGAAGCCTAACTACAGCGA-3' and antisense, 5'-AGATGAGCATTGGCAGCGAG-3'; Slug sense, 5'-GGG GAGAAGCCTTTTTCTTG-3' and antisense, 5'-TCCTCA TGTTTGTGCAGGAG-3'; GAPDH (control) sense, 5'-TGT TGCCATCAATGACCCCTT-3' and antisense, 5'-CTCCAC GACGTACTCAG CG-3'. Relative quantification was achieved by normalization of GAPDH.

Adenovirus transduction. Recombinant adenoviruses Ad- $\beta$-catenin or Ad-GFP were transduced into MDA-MB-231 cells according to the manufacturer's instructions. The expres- sion of GFP was used as a marker for monitoring transduction efficiency. After $48 \mathrm{~h}$ of transduction, the cells were used for experiments and the target gene expression was also determined by qRT-PCR and western blotting.

Immunohistochemistry (IHC) staining. The expression of molecules which were association with EMT was detected by IHC staining. Briefly, 5- $\mu \mathrm{m}$ thick tissue sections were cut and deparaffinized, and an antigen retrieval procedure was performed. Endogenous peroxidases were quenched by incubating tissue with hydrogen peroxide, followed by incubating with the primary antibody at $4{ }^{\circ} \mathrm{C}$ overnight and the HRP-labeled secondary antibody sequentially. Finally, the sections were visualized with DAB staining and imaged. 

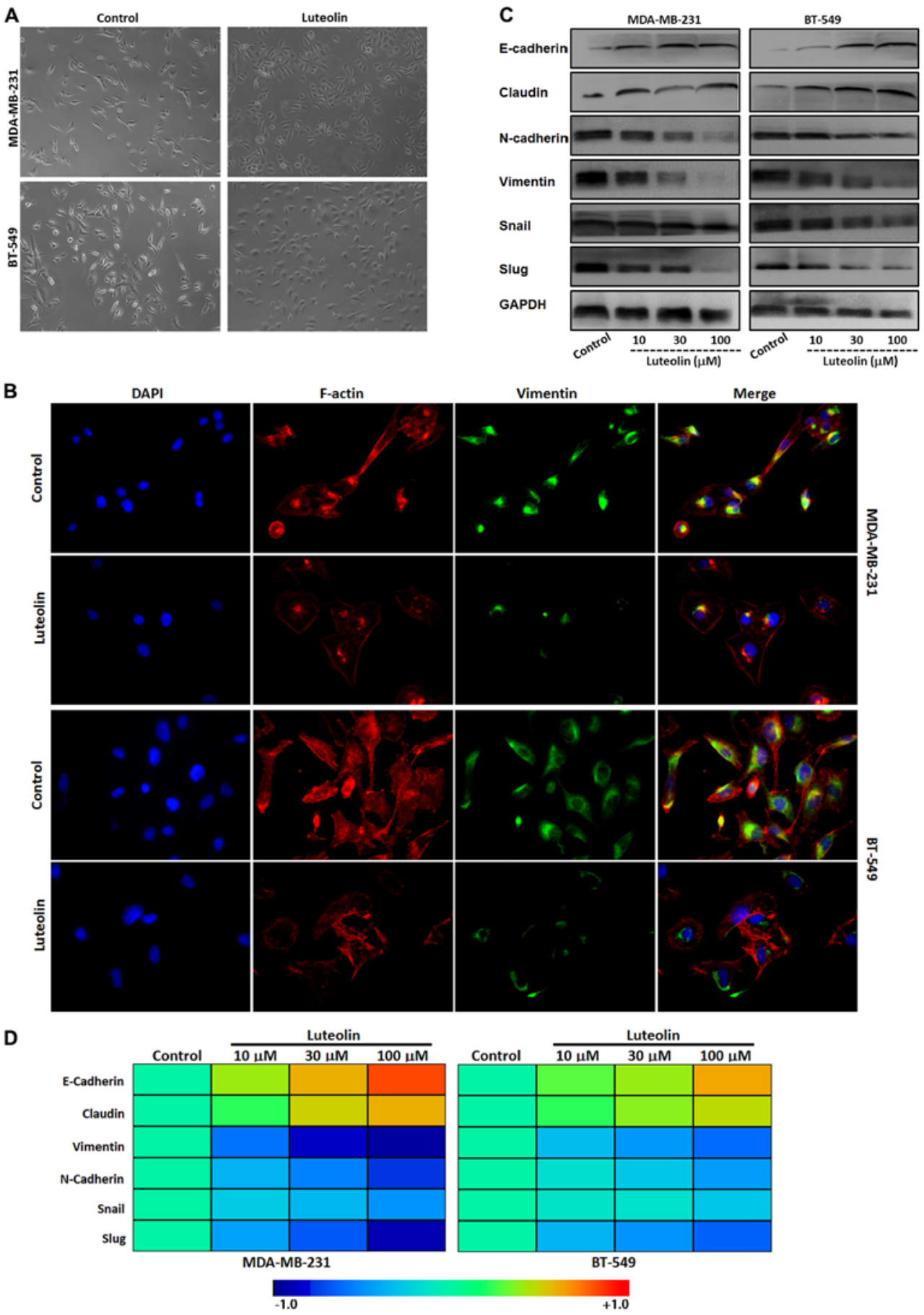

Figure 2. Luteolin reverses EMT of breast cancer cells in vitro. TNBC cell lines MDA-MB-231 and BT5-49 were pretreated with various concentrations of luteolin $(10,30$ and $100 \mu \mathrm{M})$ for the indicated time. (A) Cell morphology was evaluated by phase-contrast microscopy. (B) The cells were subjected to immunofluorescence for vimentin and F-actin. (C) The cells were subjected to western blotting for the indicated proteins. (D) The cells were subjected to qRT-PCR for the indicated mRNAs. EMT, epithelial-mesenchymal transition; TNBC, triple-negative breast cancer.

Statistical analysis. All experiments were conducted for at least three independent times. All data are expressed as mean \pm SD and the Student's t-test and one-way ANOVA analysis were used to determine significance. $\mathrm{P}<0.05$ was considered significant.

\section{Results}

Luteolin inhibits breast cancer cell migration and invasion in vitro. To determine whether luteolin influences breast cancer cell migration and invasion, wound-healing migration and Transwell invasion assays were performed to evaluate the metastasis of breast cancer cell lines MDA-MB-231 and BT5-49. Pretreatment with luteolin resulted in a concentration-dependent slowing of the wound healing ability of breast cancer cells compared with the control group (Fig. 1B and C). The Transwell invasion assay revealed a dose-dependent decrease in cell invasion in the luteolin-treated breast cancer cell lines when compared with the control group (Fig. 1D and E). 
A

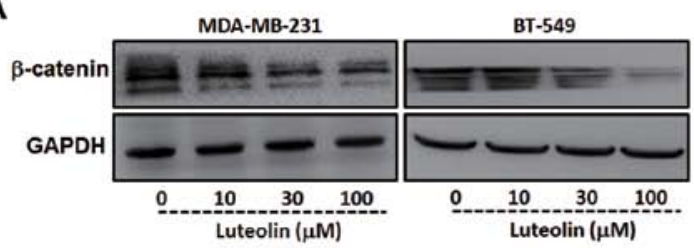

B
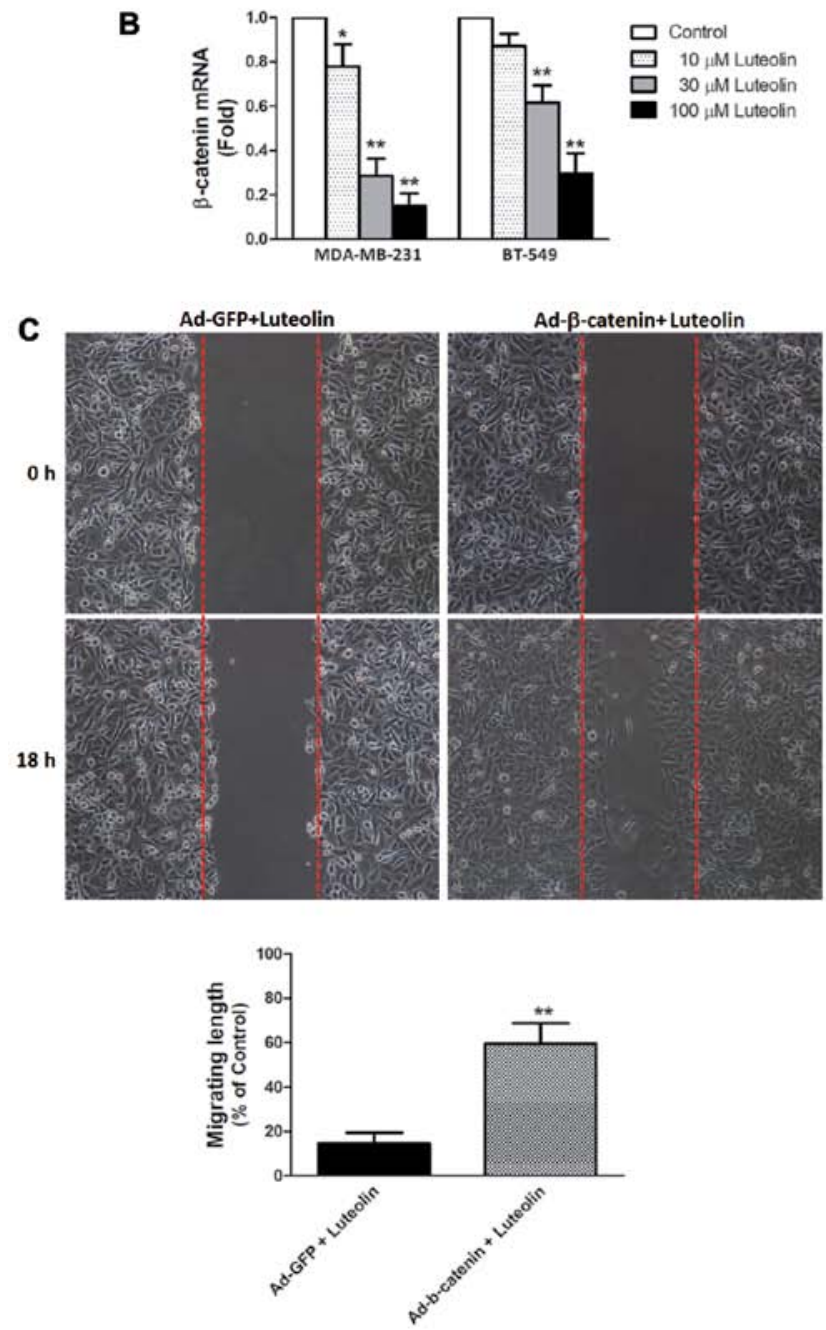
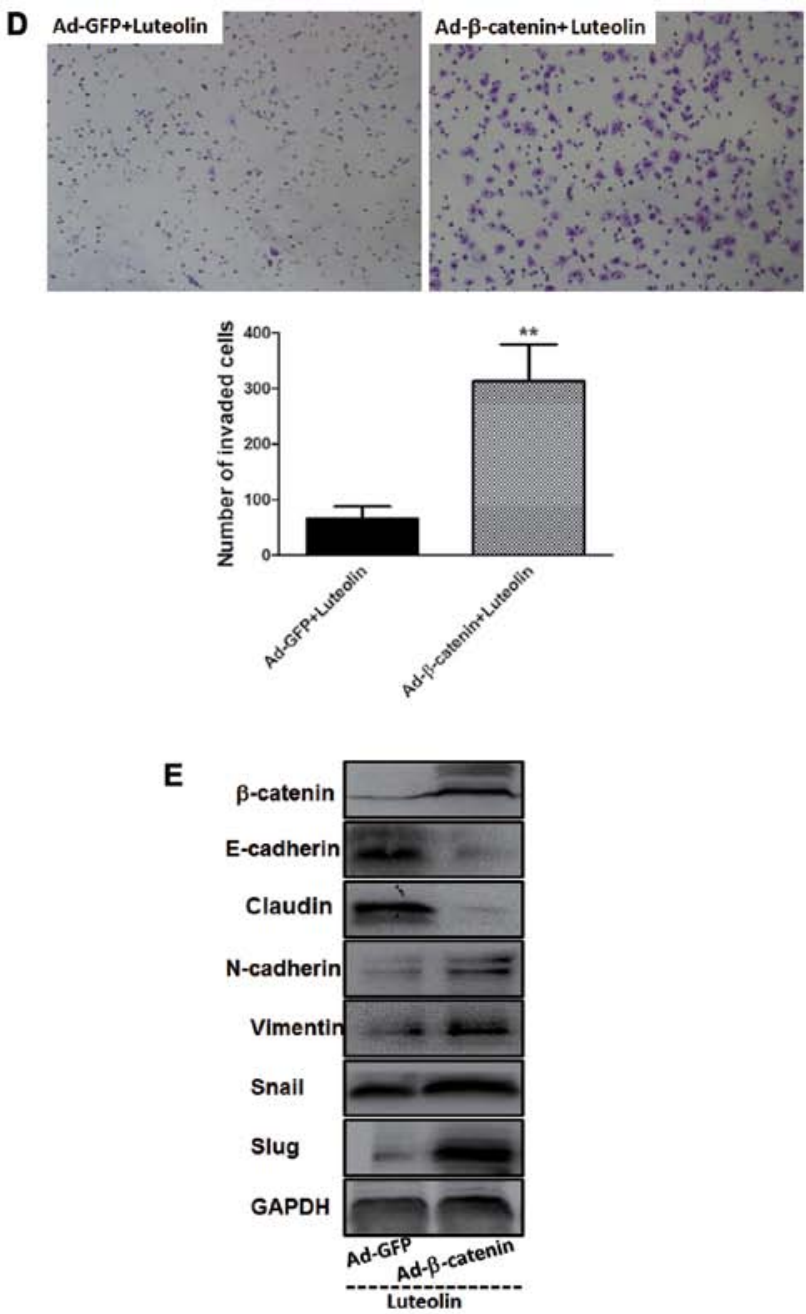

$\mathbf{F}$

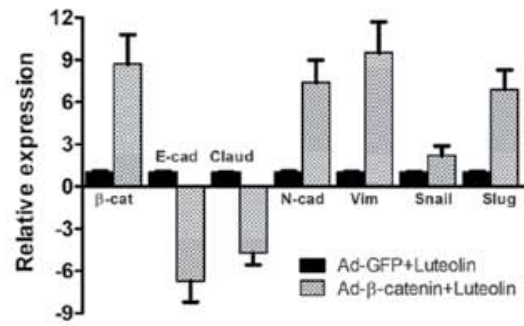

Figure 3. Luteolin downregulates $\beta$-catenin expression to reverse EMT in breast cancer cells. TNBC cell lines MDA-MB-231 and BT5-49 were pretreated with various concentrations of luteolin $(10,30$ and $100 \mu \mathrm{M})$, and the expression of (A) $\beta$-catenin protein and (B) mRNA was determined by western blotting and qRT-PCR, respectively. In the other experiment, MDA-MB-231 cells were transduced with Ad-GFP or Ad- $\beta$-catenin, and the cells were subsequently treated with PBS or luteolin $(100 \mu \mathrm{M})$. (C) The cell migration was determined by wound-healing assay. (D) The cell invasion was determined by Transwell invasion assay. (E) Protein levels and (F) mRNA expression of $\beta$-catenin and EMT-related molecules were determined as indicated. The results are shown as the mean \pm SD of three experiments; ${ }^{*} \mathrm{P}<0.05,{ }^{* *} \mathrm{P}<0.01$. EMT, epithelial-mesenchymal transition; TNBC, triple-negative breast cancer.

Luteolin reverses EMT in the MDA-MB-231 and BT5-49 cells. As previously stated, the morphology of MDA-MB231 and BT5-49 cells display a long spindle mesenchymal feature. Following treatment with luteolin, the cancer cell morphology shifted to an oval epithelial type (Fig. 2A). In agreement with this observation, IF assay showed that luteolin downregulated the mesenchymal marker vimentin and reorganized cytoskeletal protein F-actin in the cytoplasm in the two breast cancer cell lines (Fig. 2B). Western blot analysis further showed that levels of epithelial markers E-cadherin and claudin were upregulated, while mesenchymal markers $\mathrm{N}$-cadherin and vimentin with the two EMT-related transcription factors Snail and Slug, particularly Slug, were downregulated in the luteolin-treated breast cancer cells in a concentration-dependent manner (Fig. 2C). Consistently, mRNA expression analysis by qRT-PCR showed similar results with the proteins of EMT (Fig. 2D).

$\beta$-catenin is responsible for the anti-migration and antiinvasion effect of luteolin in vitro. In light of the key role of $\beta$-catenin in metastasis and EMT of cancer, we determined the expression of $\beta$-catenin by western blotting and qRT-PCR. As shown in Fig. 3A and B, compared with the control group, the two breast cancer cell lines expressed higher levels of 
A

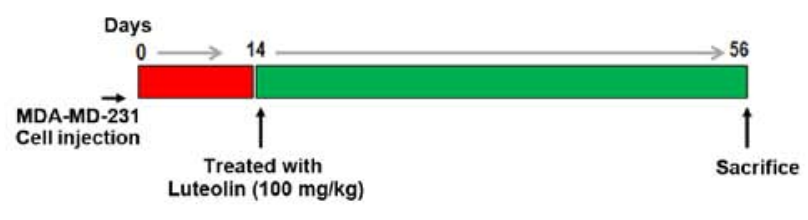

C

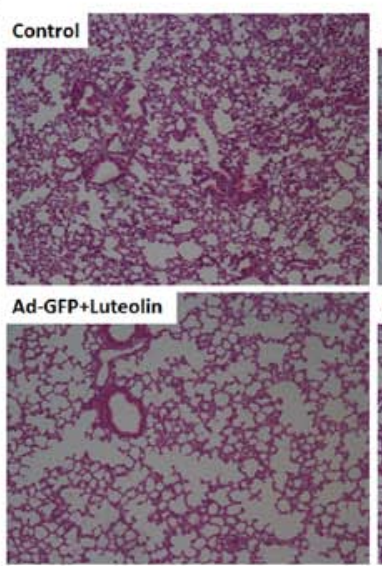

E

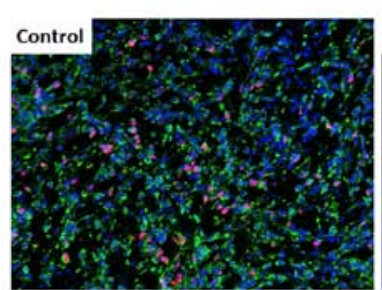

Ad-GFP+Luteolin

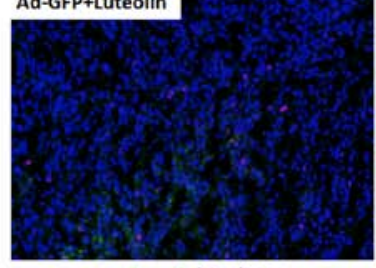

Vimentin/Slug/DAPI
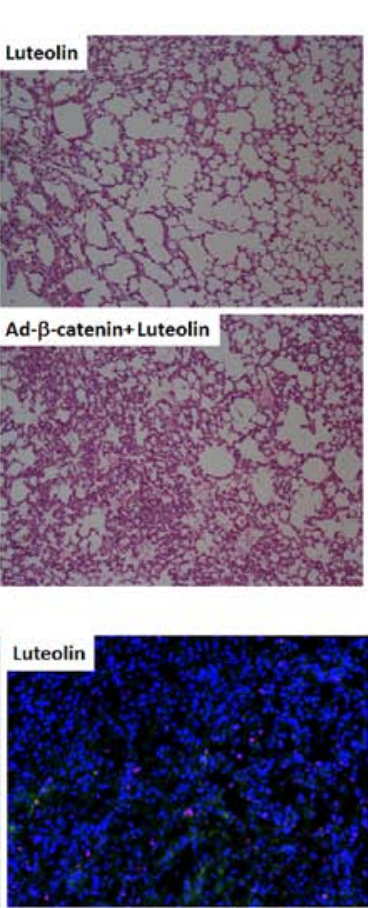

Ad- $\beta$-catenin+Luteolin

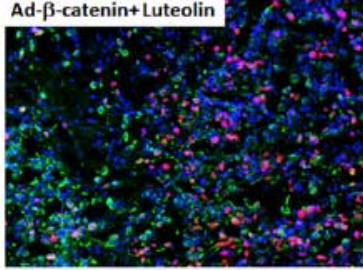

Vimentin/Slug/DAPI
B

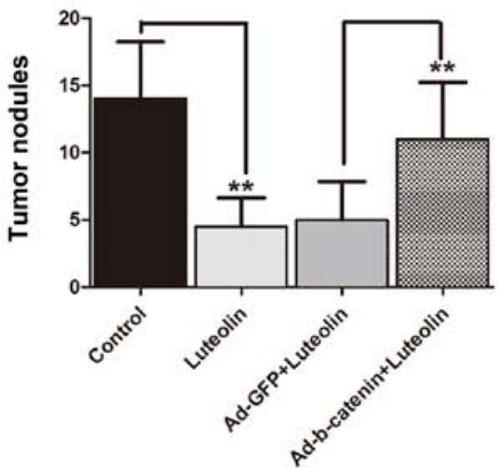

D

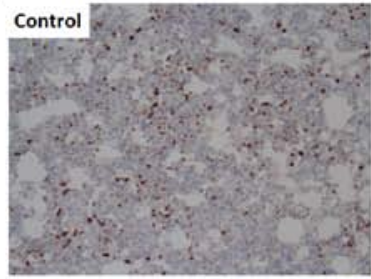

Ad-GFP+Luteolin
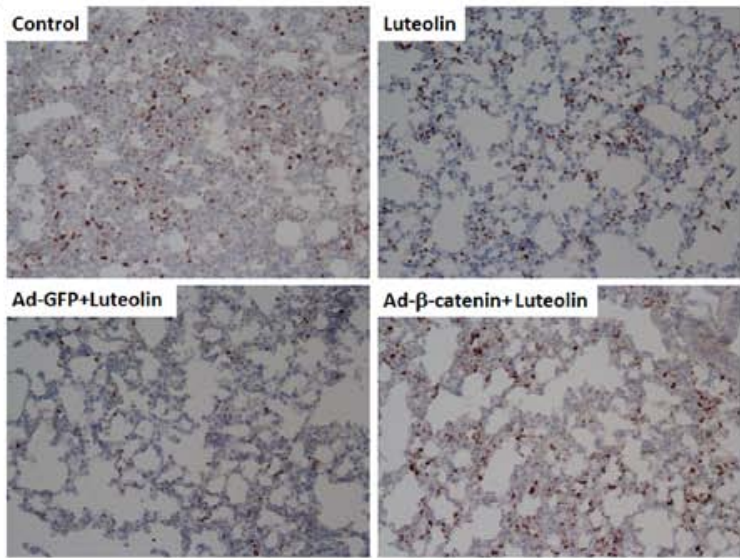

Ad- $\beta$-catenin+Luteolin

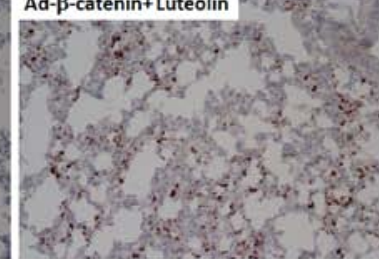

$\mathbf{F}$

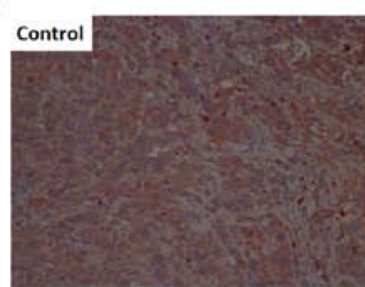

Ad-GFP+Luteolin

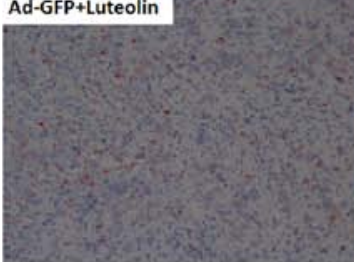

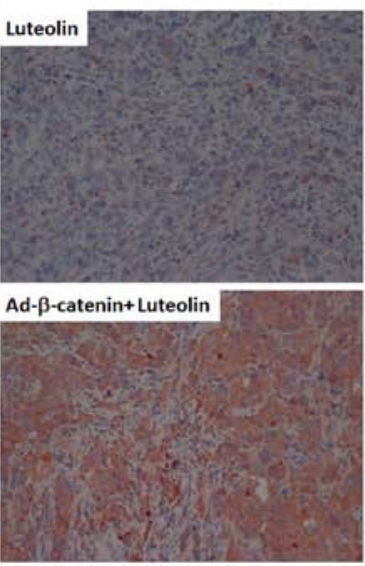

Figure 4. Luteolin inhibits the metastasis of breast cancer in vivo. (A) Xenograft tumor metastasis was established by subcutaneous injection of MDA-MB-231 cells with or without transduction of Ad-GFP or Ad- $\beta$-catenin in the presence or absence of luteolin (100 mg/kg). (B) Tumor nodules at the surface of the lung were counted. The results are shown as the mean $\pm \mathrm{SD}$ of three experiments; ${ }^{* *} \mathrm{P}<0.01$. (C) Representative images of H\&E stained sections of the metastatic nodules in the lung. (D) Representative images of Ki67-stained sections of the lung isolated from nude mice bearing breast cancer. (E) Vimentin and Slug in the primary tumors were examined by immunofluorescence. (F) $\beta$-catenin in the primary tumors was examined by immunohistochemistry.

$\beta$-catenin protein and mRNA, which were inhibited by luteolin in a dose-dependent manner. Furthermore, overexpression of $\beta$-catenin by an adenovirus vector system was carried out in the breast cancer cell line MDA-MB-231. As shown in Fig. $3 \mathrm{C}$ and D, overexpression of $\beta$-catenin abrogated the anti-migration and anti-invasion effect of luteolin in the MDA-MB-231 cells. Meantime, we found that these benefits of luteolin on the expression of EMT markers and EMT-related transcription factors were abrogated by overexpression of $\beta$-catenin (Fig. 3E and F).

Luteolin suppresses metastasis of breast cancer cells in vivo. Finally, we explore the in vivo effect of luteolin on cancer metastasis. As illustrated in Fig. 4A, using a xenograft metastasis tumor model, we found that the prominent metastatic nodules on the surface of the lung were obviously observed in the mice bearing TNBC cells. Luteolin markedly decreased the number of nodules in the lung, which were abrogated by overexpression of $\beta$-catenin (Fig. 4B). H\&E and Ki67 staining of lungs isolated from the mice receiving orthotopic transplantation displayed that luteolin markedly inhibited breast cancer cell metastases to the lung, while overexpression of $\beta$-catenin abolished the inhibitory effect of luteolin on lung metastases of breast cancer (Fig. 4C and D). Noteworthy, the levels of mesenchymal marker vimentin and transcription repressor Slug in the primary tumor tissues were 
decreased in the orthotopic tumors of the mice after luteolin treatment, while overexpression of $\beta$-catenin upregulated vimentin and Slug (Fig. 4E). In agreement with these in vivo observations, $\beta$-catenin emerged as a high expression protein in primary tumors of xenograft metastatic mice but was obviously downregulated by luteolin treatment (Fig. 4F).

\section{Discussion}

Breast cancer is a heterogeneous disease, which is classified into at least five distinct subtypes according to molecular profiles of gene expression (25). Among these subtypes, triple-negative breast cancer (TNBC), defined as estrogen receptor-negative, progesterone receptor-negative, and lack of HER-2 expression, is an aggressive metastatic and higher histological grade subtype of breast cancer (23). Due to the lack of effective targeted therapy and highly metastatic behavior, patients with TNBC have a relatively poor outcome (26). In the present study, we confirmed that luteolin efficiently suppressed the migration and invasion of TNBC cell lines MDA-MB-231 and BT-549, and inhibited the lung metastases of breast cancer xenograft tumors derived from MDA-MD-231 cells, implying that luteolin may serve as a potential drug for the treatment of cancer metastasis in TNBC.

Accumulating evidence reveals that EMT plays a critical role in invasiveness and the metastasis of breast cancer. It has been proposed that the EMT process occurs during metastatic breast cancer in which cancer cells show a fibroblast-like and spindle morphology with loss of epithelial markers E-cadherin and claudin and gain of mesenchymal markers vimentin and $\mathrm{N}$-cadherin (27). Clinical data suggest that in patients with TNBC, cancer cells display a particular mesenchymal phenotypic shift, and the expression of mesenchymal markers is associated with poor prognosis and metastatic potential $(23,28)$. Previous studies have shown that mesenchymal molecules promote migration and invasion of breast cancer cells in vitro and in vivo models, and knockout of the vimentin or $\mathrm{N}$-cadherin gene effectively attenuates breast cancer cell motility and invasion $(29,30)$. Based on the antimetastatic capacity of luteolin, we aimed to ascertain whether luteolin affects EMT of TNBC. Our present results indicated that following treatment with luteolin, TNBC cells not only acquired epithelial characteristics, but also lost mesenchymal characteristics, implying that luteolin could effectively reverse EMT in highly metastatic TNBC. This is consistent with a study describing that luteolin inhibited EMT and invasiveness in pancreatic cancer cell lines (31). Furthermore, we also analyzed the expression of two transcription factors Snail and Slug, which have been demonstrated to regulate EMT and promote tumor metastasis in breast cancer (32). The results showed that luteolin dose-dependently inhibited the expression of these transcription repressors, particularly Slug. These data confirmed that the inhibition of metastasis by luteolin involves the reversion of EMT.

Many signaling mechanisms have been implicated to be involved in the regulation of EMT and cancer metastasis, including the PI3K/Akt, MAPK/ERK and $\mathrm{Wnt} / \beta$-catenin pathways (3). Several studies have uncovered that $\mathrm{Wnt} / \beta$ catenin signaling is significantly activated in highly aggressive metastatic breast cancer cell lines and patients with TNBC, and hyperactivated $\mathrm{Wnt} / \beta$-catenin signaling is positively correlated with poor clinical prognosis $(33,34)$. In canonical Wnt $/ \beta$-catenin signaling, $\beta$-catenin plays a central role. Activation of Wnt signaling leads to the stabilization and subsequent nuclear translocation of $\beta$-catenin, which induces expression of transcriptional repressors such as Snail, triggering EMT that is linked to cancer metastasis $(34,35)$. Inhibition or silencing of $\beta$-catenin markedly inhibits the expression of EMT-related transcriptional factors Snail and Slug, which lead to the expression of epithelial markers E-cadherin, resulting in reversal of EMT and alleviation of the metastasis of breast cancer $(36,37)$. Thereby, $\beta$-catenin may become a key target for treating metastatic cancer. Notably, a previous study showed that luteolin inhibited cell proliferation and tumor growth in experimental colon carcinogenesis by inhibiting the $\beta$-catenin signaling pathway (38). In the present study, using in vitro and in vivo metastasis breast cancer models, we first found that $\beta$-catenin expression was dose-dependently reduced by luteolin in two TNBC cell lines, and we further found that luteolin markedly downregulated $\beta$-catenin in primary tumor tissues of the xenografts, which coincided with the abilities of luteolin on metastasis and EMT of breast cancer. Hence, we deduced that the antimetastatic affect of luteolin may be mediated by $\beta$-catenin signaling. In order to verify the hypothesis, ectopic expression of $\beta$-catenin was determined before luteolin treatment in vitro and in vivo metastasis experiments. We found that these beneficial effects of luteolin were abrogated by overexpression of $\beta$-catenin, indicating that downregulation of $\beta$-catenin expression may mediate the inhibitory effects of luteolin on metastasis and EMT of TNBC.

Taken together, our experimental data indicated that luteolin exerted a potent therapeutic effect on invasion and metastasis of TNBC, which may be involved in the reversal of EMT by downregulation of $\beta$-catenin. These findings suggest that luteolin may be applied as a potential candidate treatment for the prevention and intervention of metastatic breast cancer.

\section{Acknowledgements}

The present study was supported by grants from the National Natural Science Foundation of China (no. 81472475). We thank Dr Jianfei Guo, James Winkle College of Pharmacy, University of Cincinnati, $\mathrm{OH}$ for the discussion and proofreading of the manuscript.

\section{References}

1. DeSantis CE, Fedewa SA, Goding Sauer A, Kramer JL, Smith RA and Jemal A: Breast cancer statistics, 2015: Convergence of incidence rates between black and white women. CA Cancer J Clin 66: 31-42, 2016.

2. Arnedos M, Vicier C, Loi S, Lefebvre C, Michiels S, Bonnefoi H and Andre F: Precision medicine for metastatic breast cancer - limitations and solutions. Nat Rev Clin Oncol 12: 693-704, 2015.

3. Lamouille S, Xu J and Derynck R: Molecular mechanisms of epithelial-mesenchymal transition. Nat Rev Mol Cell Biol 15: 178-196, 2014.

4. Thiery JP, Acloque H, Huang RY and Nieto MA: Epithelialmesenchymal transitions in development and disease. Cell 139: 871-890, 2009.

5. Zeisberg M and Neilson EG: Biomarkers for epithelial-mesenchymal transitions. J Clin Invest 119: 1429-1437, 2009 
6. Baranwal S and Alahari SK: Molecular mechanisms controlling E-cadherin expression in breast cancer. Biochem Biophys Res Commun 384: 6-11, 2009.

7. Peinado H, Olmeda D and Cano A: Snail, Zeb and bHLH factors in tumour progression: An alliance against the epithelial phenotype? Nat Rev Cancer 7: 415-428, 2007.

8. Mani SA, Guo W, Liao MJ, Eaton EN, Ayyanan A, Zhou AY, Brooks M, Reinhard F, Zhang CC, Shipitsin M, et al: The epithelial-mesenchymal transition generates cells with properties of stem cells. Cell 133: 704-715, 2008.

9. Singh A and Settleman J: EMT, cancer stem cells and drug resistance: An emerging axis of evil in the war on cancer. Oncogene 29: 4741-4751, 2010.

10. Polyak K and Weinberg RA: Transitions between epithelial and mesenchymal states: Acquisition of malignant and stem cell traits. Nat Rev Cancer 9: 265-273, 2009.

11. McConkey DJ, Choi W, Marquis L, Martin F, Williams MB, Shah J, Svatek R, Das A, Adam L, Kamat A, et al: Role of epithelial-to-mesenchymal transition (EMT) in drug sensitivity and metastasis in bladder cancer. Cancer Metastasis Rev 28: 335-344, 2009.

12. Xu L, Zhang L, Hu C, Liang S, Fei X, Yan N, Zhang Y and Zhang F: WNT pathway inhibitor pyrvinium pamoate inhibits the self-renewal and metastasis of breast cancer stem cells. Int J Oncol 48: 1175-1186, 2016.

13. Ross JA and Kasum CM: Dietary flavonoids: Bioavailability, metabolic effects, and safety. Annu Rev Nutr 22: 19-34, 2002.

14. Lin Y, Shi R, Wang X and Shen HM: Luteolin, a flavonoid with potential for cancer prevention and therapy. Curr Cancer Drug Targets 8: 634-646, 2008.

15. Chen CY, Peng WH, Tsai KD and Hsu SL: Luteolin suppresses inflammation-associated gene expression by blocking NF-kappaB and AP-1 activation pathway in mouse alveolar macrophages. Life Sci 81: 1602-1614, 2007.

16. Chung JG, Hsia TC, Kuo HM, Li YC, Lee YM, Lin SS and Hung CF: Inhibitory actions of luteolin on the growth and arylamine $\mathrm{N}$-acetyltransferase activity in strains of Helicobacter pylori from ulcer patients. Toxicol In Vitro 15: 191-198, 2001.

17. Du Y, Feng J, Wang R, Zhang H and Liu J: Effects of flavonoids from Potamogeton crispus L. on proliferation, migration, and invasion of human ovarian cancer cells. PLoS One 10: e0130685, 2015.

18. Ma L, Peng H, Li K, Zhao R, Li L, Yu Y, Wang X and Han Z: Luteolin exerts an anticancer effect on NCI-H460 human non-small cell lung cancer cells through the induction of Sirt1-mediated apoptosis. Mol Med Rep 12: 4196-4202, 2015.

19. Shi R, Huang Q, Zhu X, Ong YB, Zhao B, Lu J, Ong CN and Shen HM: Luteolin sensitizes the anticancer effect of cisplatin via c-Jun $\mathrm{NH}_{2}$-terminal kinase-mediated p53 phosphorylation and stabilization. Mol Cancer Ther 6: 1338-1347, 2007.

20. Yang MY, Wang CJ, Chen NF, Ho WH, Lu FJ and Tseng TH: Luteolin enhances paclitaxel-induced apoptosis in human breast cancer MDA-MB-231 cells by blocking STAT3. Chem Biol Interact 213: 60-68, 2014

21. Tu SH, Ho CT, Liu MF, Huang CS, Chang HW, Chang CH, $\mathrm{Wu} \mathrm{CH}$ and Ho YS: Luteolin sensitises drug-resistant human breast cancer cells to tamoxifen via the inhibition of cyclin E2 expression. Food Chem 141: 1553-1561, 2013.

22. Cook MT, Liang Y, Besch-Williford C, Goyette S, Mafuvadze B and Hyder SM: Luteolin inhibits progestin-dependent angiogenesis, stem cell-like characteristics, and growth of human breast cancer xenografts. Springerplus 4: 444, 2015.

23. Lehmann BD, Bauer JA, Chen X, Sanders ME, Chakravarthy AB, Shyr Y and Pietenpol JA: Identification of human triple-negative breast cancer subtypes and preclinical models for selection of targeted therapies. J Clin Invest 121: 2750-2767, 2011.
24. Zhao C, Qiao Y, Jonsson P, Wang J, Xu L, Rouhi P, Sinha I, Cao Y, Williams C and Dahlman-Wright K: Genome-wide profiling of AP-1-regulated transcription provides insights into the invasiveness of triple-negative breast cancer. Cancer Res 74: 3983-3994, 2014

25. Voduc KD, Cheang MCU, Tyldesley S, Gelmon K, Nielsen TO and Kennecke H: Breast cancer subtypes and the risk of local and regional relapse. J Clin Oncol 28: 1684-1691, 2010.

26. Foulkes WD, Smith IE and Reis-Filho JS: Triple-negative breast cancer. N Engl J Med 363: 1938-1948, 2010.

27. Thiery JP: Epithelial-mesenchymal transitions in tumour progression. Nat Rev Cancer 2: 442-454, 2002

28. Carey L, Winer E, Viale G, Cameron D and Gianni L: Triplenegative breast cancer: Disease entity or title of convenience? Nat Rev Clin Oncol 7: 683-692, 2010.

29. Vuoriluoto K, Haugen H, Kiviluoto S, Mpindi JP, Nevo J, Gjerdrum C, Tiron C, Lorens JB and Ivaska J: Vimentin regulates EMT induction by Slug and oncogenic $\mathrm{H}$-Ras and migration by governing Axl expression in breast cancer. Oncogene 30: $1436-1448,2011$.

30. Chung S, Yao J, Suyama K, Bajaj S, Qian X, Loudig OD, Eugenin EA, Phillips GR and Hazan RB: N-cadherin regulates mammary tumor cell migration through Akt3 suppression. Oncogene 32: 422-430, 2013.

31. Huang X, Dai S, Dai J, Xiao Y, Bai Y, Chen B and Zhou M: Luteolin decreases invasiveness, deactivates STAT3 signaling, and reverses interleukin- 6 induced epithelial-mesenchymal transition and matrix metalloproteinase secretion of pancreatic cancer cells. Onco Targets Ther 8: 2989-3001, 2015.

32. de Herreros AG, Peiró S, Nassour M and Savagner P: Snail family regulation and epithelial mesenchymal transitions in breast cancer progression. J Mammary Gland Biol Neoplasia 15: $135-147,2010$.

33. Dey N, Barwick BG, Moreno CS, Ordanic-Kodani M, Chen Z, Oprea-Ilies G, Tang W, Catzavelos C, Kerstann KF, Sledge GW Jr, et al: Wnt signaling in triple negative breast cancer is associated with metastasis. BMC Cancer 13: 537, 2013.

34. Geyer FC, Lacroix-Triki M, Savage K, Arnedos M, Lambros MB, MacKay A, Natrajan R and Reis-Filho JS: $\beta$-Catenin pathway activation in breast cancer is associated with triple-negative phenotype but not with CTNNB1 mutation. Mod Pathol 24: 209-231, 2011.

35. Sánchez-Tilló E, de Barrios O, Siles L, Cuatrecasas M, Castells A and Postigo A: $\beta$-catenin/TCF4 complex induces the epithelialto-mesenchymal transition (EMT)-activator ZEB1 to regulate tumor invasiveness. Proc Natl Acad Sci USA 108: 19204-19209, 2011.

36. Wu ZQ, Li XY, Hu CY, Ford M, Kleer CG and Weiss SJ: Canonical Wnt signaling regulates Slug activity and links epithelial-mesenchymal transition with epigenetic Breast Cancer 1, Early Onset (BRCA1) repression. Proc Natl Acad Sci USA 109: 16654-16659, 2012.

37. Yook JI, Li XY, Ota I, Hu C, Kim HS, Kim NH, Cha SY, Ryu JK, Choi YJ, Kim J, et al: A Wnt-Axin2-GSK3beta cascade regulates Snail1 activity in breast cancer cells. Nat Cell Biol 8: 1398-1406, 2006.

38. Ashokkumar P and Sudhandiran G: Luteolin inhibits cell proliferation during Azoxymethane-induced experimental colon carcinogenesis via Wnt $/ \beta$-catenin pathway. Invest New Drugs 29: 273-284, 2011. 